\title{
Resolution of coronary 'mother'- 'daughter' configurations by multi-artery FFR method - a comparison with basic FFR method
}

Background: A sizable coronary artery and its small side-branch are usually termed 'mother''daughter' configuration. In the intermediate stenosis severity range resolution of such configurations might not be trivial. Numerical simulation by the novel Multi-artery FFR method will be presented. Such configurations can be also resolved by the basic FFR method. The objective is a comparison between the two methods and exhibition of the effect of stenosis-stenosis interactions.

Methods: A 'mother' artery stenotic at locations preceding and following the split-off point of stenotic 'daughter' artery is considered. Multi-artery FFR method is applied to an equivalent 3-artery configuration. The difference between a stenotic single artery in a virtual stand-alone position (with FFR=FFR ${ }^{\text {true }}$ ) and the same artery within a configuration of interacting stenotic arteries with a different actual FFR (denoted FFR ${ }^{\text {real }}$ ) is discussed.

Results: An artery in seemingly acceptable condition (FFR $\left.{ }^{\text {true }} \sim 0.93\right)$ may have unacceptable actual FFR (FFR ${ }^{\text {real }} 0.65$ ) requiring revascularization (see example in article). The Multi-artery FFR method has the advantage of predicting post-revascularization FFR ${ }^{\text {real }}$ of each artery from initial intracoronary pressure data whereas basic FFR method can yield post-revascularization FFR real of each artery only after revascularizations are actually made.

Conclusions: Multi-artery FFR method mathematics used by the $\mathrm{PCl}$ practitioner is simple. It can be used in real time to obtain the current status of stenotic 'mother'-'daughter' configuration and its final optimal resolution. The basic single-artery FFR method is also applicable in such a scenario. A comparison between the two methods favors the Multi-artery FFR method essentially for its capability to predict revascularization outcomes.

Keywords: Percutaneous coronary intervention (PCI) - Fractional flow reserve (FFR) - Multiartery fractional flow reserve

\section{Introduction}

The decision of whether to perform a revascularization or leave a coronary stenosis to a conservative medical treatment may be of very far reaching medical as well as financial consequences to the patient. An incorrect decision to go ahead with a revascularization can expose the patient to risks. The risks of a full scale coronary artery bypass graft (CABG) operation are well known. As for stenting the artery at the location of the stenosis, it has its own risks. Though in-stent restenosis (ISR) has been substantially diminished by the use of drug-eluting stents (DES), it has not been eradicated. Within a period of a year or two, in cases of simple lesions and without particular risk factors, the ISR rate is usually less than 5\% [1]. In cases of complex lesions and additional risk factors however, double digit rates are not uncommon [2]. The sudden incidence of stent thrombosis (ST) is around $1 \%[3]$. In each of the two extreme
Ilan A. Yaeger*

18 Karkom Street, Karmiel, Israel

*Author for correspondence:

Tel.: +972-4-9883669

Email: ilan.yaeger41@gmail.com

Received date: June 05, 2018

Accepted date: June 19, 2018

Published date: June 25, 2018 
cases, the very low and the very high stenosis severity ranges, the decision from which the patient can benefit is obvious from visual inspection and can be readily made. It is in the intermediate stenosis severity range (30\%-70\% diameter stenosis) where visual stenosis severity assessment can be difficult.

Despite the introduction of more advanced FFRoriented stenosis severity assessment methods, in more than $70 \%$ of percutaneous coronary intervention (PCI) procedures the assessments of the stenoses in the epicardial arteries are of basic angiographic nature and are based on the PCI visual data [4].

In this article stenotic 'mother'-'daughter' coronary configurations will be assessed and only localized discrete and diffuse stenoses that do not involve the whole length of the artery will be considered. Also, for simplicity, it will be assumed that collaterals are not involved with the epicardial arterial configurations that are under consideration in the article. Naturally, dealing with an FFR method, it will be further assumed that the intracoronary pressures are measured when the micro vascular resistances are minimal and stable.

The basic FFR method is superior to visual inspection of angiographic characteristics of stenotic epicardial arteries, as was shown in the PCI procedures of the famous FAME statistical study [5,6]. However, this method cannot handle cases where inter-arterial interactions take place. In the FAME study only stenosis-free LMCA cases were considered hence the aortic pressure was maintained all the way to the LAD and LCx arteries. The LAD and LCx arteries (as well as the RCA) were therefore non-interacting, the flow in each of them did not affect the flow in other arteries and each could be assessed separately as in a single vessel disease (SVD) case. If in a 'mother'-'daughter' configuration, the 'daughter' artery is sufficiently small, it does not affect the flow in the sizable 'mother' artery and the latter can be approximated as a single artery. Under such conditions the basic single-artery FFR approach can be used, in order to resolve the stenotic 'mother'-'daughter' configuration. If by the basic FFR approach a revascularization of the stenotic sizable artery is required, it is possible to tell by the pullback technique which stenosis has priority for treatment. However, following the revascularization the pullback technique needs to be used again in order to find out if the other stenosis in the 'mother' artery needs to be treated too (and to check on the new FFR of Artery 3 in Figure 1). This subjects the patient again to the uncomfortable hyperemia condition. On the other

\section{Equivalent 3-artery configuration}

Pressure-Resistance-Flow scheme

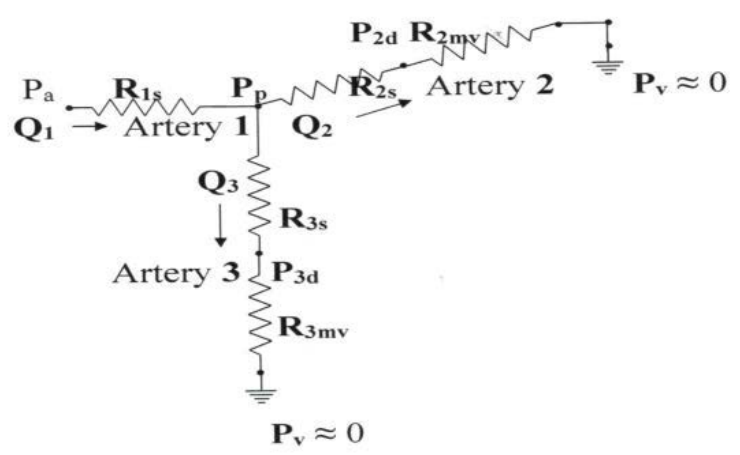

Figure 1: First subscript of resistance $R$ indicates the artery number (1,2 or 3 ) and the second subscript indicates the type of resistance (s-stenotic, mv-microvascular). $\mathrm{Pa}$ and $\mathrm{Pv}$ are the mean aortic pressure and the nearly zero pressure of the venous bed respectively. Pressures $P$ at other points are defined within the text. Qi is the blood flow (volume units per unit of time) in Artery $i(i=1,2,3)$. In the usual analysis of a stenotic 3 -artery configuration, Artery 1 is functionally the conductance artery and Arteries 2 and 3 are the end arteries. In the analysis of a stenotic 'mother'-'daughter' configuration, the combined Arteries 1 and 2 in the equivalent 3-artery configuration are the sizable 'mother' artery whereas Artery 3 represents the small 'daughter' artery.

hand, if the Multi-artery FFR method is used, this very same goal can be reached by measuring intracoronary pressures only once. Note however that the post revascularization repeated pullback use in the basic FFR method offers a chance to compare the new measured FFR values with the ones calculated by the Multiartery FFR method in its exploration stage of future revascularization options.

The Multi-artery FFR method does take into account inter-arterial interactions (usually termed stenosisstenosis interactions) in epicardial stenotic arterial configurations $[7,8]$. In a stand-alone position, the stenosis severity of a single stenotic artery is assessed by the basic single-artery FFR method that yields the FFR of the artery (denoted FFR ${ }^{\text {true}}$ ). When the stenotic artery under consideration is part of a configuration of stenotic arteries, its flow is affected by the flow in other arteries of the configuration and the actual FFR of the artery is no longer FFR ${ }^{\text {rue }}$. The Multi-artery FFR method is then used to calculate the actual FFR (denoted FFR ${ }^{\text {real }}$ ) of the artery. FFR ${ }^{\text {real }}$ is the ratio between the actual flow $\mathrm{Q}^{(s)}$ through the stenotic artery and the presumable flow $Q^{(o)}$ through the artery when all the arteries are virtually stenosis-free. Usually $\mathrm{FFR}^{\text {real }} \neq \mathrm{FFR}^{\text {rue }}$ because the FFR 
of an artery that shares an arterial configuration with other arteries is influenced by interactions with other members of the configuration $[7,8]$. FFR ${ }^{\text {true }}$ may be regarded in such circumstances as the intrinsic FFR of the stenotic artery. For instance, if an artery undergoes revascularization, the $\mathrm{FFR}^{\text {true }}$ of the artery is taken to be equal to 1.00 (though in reality stenting may sometimes yield a slightly lower value).

Despite the somewhat lengthy mathematics involved in the development of the Multi-artery FFR method [7-9], the final formulas for $\mathrm{FFR}^{\text {real }}$ of the arteries placed into the hands of the PCI practitioner using this method are simple and can be used by him/her in real time during the PCI procedure. Furthermore, there are also simple formulas for $\mathrm{FFR}^{\text {real }}$ of possible revascularizations that can be carried out in PCI real time when seeking optimal resolution of the stenotic configuration [7-9]. This will be demonstrated in this article too.

\section{Methods}

The 'mother'-daughter' configuration cases in the present paragraph will be generalized to allow stenosis in the 'mother' artery both at a location preceding the split-off point of the stenotic 'daughter' artery and at a location following the split-off point. The cases considered in the paragraph will be only the ones in which the primary driving pressure in the configuration (proximal pressure of 'mother' artery) is an aortic or nearly aortic pressure. This implies that there is no stenosis in LMCA or in any epicardial artery leading from the aorta to the beginning of the 'mother' artery. Also, in the calculations the geometrical viscous resistances of the stenosis-free segments of the epicardial arteries are assumed negligible compared to resistances of arterial stenoses and to relevant microvascular resistances.

In order to apply the Multi-artery FFR method to 'mother'-'daughter' configurations of the kind described above, an equivalent stenotic 3-artery configuration given schematically in Figure $\mathbf{1}$ will be considered. Going by the scheme of Figure 1, Artery $\mathbf{1}$ represents the stenotic initial part of the 'mother' artery all the way to the split-off point. Artery $\mathbf{2}$ represents the stenotic remainder of the 'mother' artery, from the 'daughter' split-off point onward. The stenotic 'daughter' artery is represented by Artery 3 of Figure $\mathbf{1}$.

For the convenience of the readers, the Multi-artery FFR formulas giving the current values of the FFR ${ }^{\text {true }}$ and FFR $^{\text {real }}$ of each of the arteries $\mathbf{i}(\mathbf{i}=1,2,3)$ of the configuration in Figure $\mathbf{1}$ in terms of the intracoronary pressures $\mathrm{P}_{\mathrm{a}}, \mathbf{P}_{\mathbf{p}}, \mathbf{P}_{\mathbf{2 d}}$ and $\mathbf{P}_{\mathbf{3 d}}$ [9] will be given here:

$$
\operatorname{FFR}^{\text {true }}(1)=1 /\left\{\left[\left(\mathrm{P}_{\mathrm{a}}-\mathbf{P}_{\mathrm{p}}\right) \cdot(1+\delta)\right] /\left(\mathrm{P}_{3 \mathrm{~d}}+\delta \cdot \mathrm{P}_{2 \mathrm{~d}}\right)+1\right\}
$$

$$
\begin{aligned}
& \operatorname{FFR}^{\text {rrue }}(2)=\mathbf{P}_{2 \mathbf{d}} / \mathbf{P}_{\mathbf{p}} \\
& \operatorname{FFR}^{\text {true }}(3)=\mathbf{P}_{3 \mathrm{~d}} / \mathbf{P}_{\mathbf{p}} \\
& \operatorname{FFR}^{\text {real }}(1)=\left(\mathbf{P}_{3 \mathrm{~d}}+\delta \cdot \mathbf{P}_{2 \mathrm{~d}}\right) /\left[\mathrm{P}_{\mathrm{a}} \cdot(1+\delta)\right] \\
& \operatorname{FFR}^{\text {real }}(2)=\mathbf{P}_{2 \mathrm{~d}} / \mathrm{P}_{\mathrm{a}} \\
& \operatorname{FFR}^{\text {real }}(3)=\mathbf{P}_{3 \mathrm{~d}} / \mathrm{P}_{\mathrm{a}}
\end{aligned}
$$

Note that $\delta$ is the ratio of the microvascular resistances:

$$
\delta=\mathrm{R}_{3 \mathrm{mv}} / \mathrm{R}_{2 \mathrm{mv}}
$$

It should be stressed that with a sizable 'mother' artery and a small 'daughter' artery, such a configuration clearly belongs to the $\delta$ high limit class, $1<<\delta$ [8]. Mathematically, the formulas pertaining to this case can be obtained by calculating the appropriate expressions in the limit $\delta \rightarrow \infty$. Practically the range of $\delta$ here is $1<\delta<10$ [8]. In 'mother'-'daughter' configurations for which $10<\delta$, usually the 'daughter' artery is already so tiny that it is contraindicated for PCI.

Numerically the resolution of the 'mother'-'daughter' configuration by the Multi-artery FFR method is carried out as following:

First the FFR ${ }^{\text {true }}$ and FFR $^{\text {real }}$ values of each artery of the equivalent 3-artery configuration in Figure $\mathbf{1}$ are obtained from the various measurable intracoronary pressures (expressions (1)-(6)). Note however that FFR $^{\text {true }}$ and FFR ${ }^{\text {real }}$ of Artery $\mathbf{1}$ are dependent on $\delta$, therefore expressions (1) and (4) should be replaced by the following expressions [8]:

$$
\begin{aligned}
& \operatorname{FFR}^{\text {rue }}(1)=1 /\left[\left(\mathrm{P}_{\mathrm{a}}-\mathbf{P}_{\mathrm{p}}\right) / \mathbf{P}_{2 \mathrm{~d}}+1\right] \\
& 1<<\delta \\
& \operatorname{FFR}^{\text {real }}(1)=\mathbf{P}_{2 \mathrm{~d}} / \mathrm{P}_{\mathrm{a}} \\
& 1<<\delta
\end{aligned}
$$

From the FFR $^{\text {real }}$ of the three arteries of the equivalent 3-artery stenotic configuration one can tell if a revascularization of an artery is required or not. A revascularization of an artery, if fully successful, transforms the current $\mathrm{FFR}^{\text {true }}$ into $\mathrm{FFR}^{\text {true }}=1.00$ for that artery. One therefore should express the FFR ${ }^{\text {real }}$ of each artery as a function of FFR ${ }^{\text {true }}$ of the arteries of the equivalent configuration in order to see the effect of a revascularization, if needed (see Example\#1 and Example\#2). 
One has [9]:

$$
\begin{aligned}
& \mathrm{FFR}^{\text {real }}(1) \approx\left[\mathrm{FFR}^{\text {true }}(1) \cdot \mathrm{FFR}^{\text {true }}(2)\right] / \\
& {\left[\operatorname{FFR}^{\text {true }}(1)+\operatorname{FFR}^{\text {true }}(2)-\mathrm{FFR}^{\text {true }}(1) \cdot \mathrm{FFR}^{\text {true }}(2)\right]} \\
& 1<<\delta
\end{aligned}
$$

$$
\operatorname{FFR}^{\text {real }}(2) \approx\left[\operatorname{FFR}^{\text {true }}(1) \cdot \operatorname{FFR}^{\text {true }}(2)\right] /
$$$$
\left[\operatorname{FFR}^{\text {true }}(1)+\operatorname{FFR}^{\text {true }}(2)-\text { FFR }^{\text {true }}(1) \cdot \operatorname{FFR}^{\text {true }}(2)\right]
$$

$$
1<\delta
$$

$\operatorname{FFR}^{\text {real }}(3) \approx\left[\operatorname{FFR}^{\text {true }}(1) \cdot \operatorname{FFR}^{\text {true }}(3)\right] /$

$\left[\operatorname{FFR}^{\text {true }}(1)+\operatorname{FFR}^{\text {true }}(2)-\right.$ FFR $^{\text {true }}(1) \cdot$ FFR $\left.^{\text {true }}(2)\right]$

$$
1<<\delta
$$

Note that $\operatorname{FFR}^{\text {real }}(1), \operatorname{FFR}^{\text {real }}(2)$ and $\operatorname{FFR}^{\text {real }}(3)$ expressions for the 'mother'-'daughter' configuration have a common denominator. This can speed-up realtime calculations during the PCI procedure.

It should be noted that the advantage of applying the Multi-artery FFR method to a stenotic coronary configuration is that the intracoronary pressures are measured only once and then the status of the configuration (FFR ${ }^{\text {rue }}$ and $\mathrm{FFR}^{\text {real }}$ of each artery) as well as the outcomes of optional future revascularizations can be calculated.

However, in the 'mother'-'daughter' configuration in this article the 'daughter' artery is small and has no effect on the flow in the sizable 'mother' artery. Therefore the 'mother' artery can be regarded to a very good approximation as a single artery with two stenoses and the whole 'mother'-daughter' stenotic configuration can be also resolved by the application of the basic single-artery FFR method. By the basic FFR method, under such circumstances the FFR of the 'mother' artery is (Figure 1):

$$
\operatorname{FFR}^{\text {real }}\left(\text { 'mother') } \approx \mathrm{FFR}^{\text {true }} \text { ('mother') } \approx \mathbf{P}_{2 \mathrm{~d}} / \mathrm{P}_{\mathrm{a}}\right.
$$

If the FFR of the 'mother' artery indicates that a revascularization of the 'mother' artery is required, the first pullback round of the basic FFR method in the 'mother' artery can point to the stenosis that needs to be treated first. It's the stenosis over which the pressure drop is higher. The pressure drop $\Delta \mathbf{P}_{1}$ over the first stenosis in the 'mother' artery is (Figure $\mathbf{1}$ ):

$$
\Delta \mathbf{P}_{1}=\mathrm{P}_{\mathrm{a}}-\mathbf{P}_{\mathbf{P}}
$$

The pressure drop $\Delta \mathbf{P}_{2}$ over the second stenosis is

$$
\Delta \mathbf{P}_{2}=\mathbf{P}_{\mathbf{P}}-\mathbf{P}_{2 \mathrm{~d}}
$$

Note that after the first revascularization of the 'mother' artery, all the intracoronary pressures change (except the mean aortic pressure) and in the basic single-artery FFR method it is necessary to run a second pullback round (also in the side branch) and measure them again in order to decide (by the new $\mathrm{FFR}^{\text {real }}$ values) if a second revascularization of the 'mother' artery and/or a revascularization of the 'daughter' artery are needed. This again offers a chance to compare the new FFR $^{\text {real }}$ values of the arteries to the ones calculated in the Multi-artery FFR method while exploring numerically the various revascularization options.

\section{Results}

In this paragraph the 'mother'-'daughter' configuration consists of a sizable 'mother' artery with a stenosis preceding the split-off point of a small stenotic side-branch and another stenosis in the 'mother' artery following the split-off point. As indicated, due to the difference in size of the 'mother' and 'daughter' arteries, $\delta$ of the Multi-artery FFR method should be taken in the mathematical limit $1<\delta$ [8]. Some comments of comparison to the single-artery FFR approach will be made within the paragraph.

In the first example we'll consider stenotic arteries with FFR $^{\text {real }}$ numerical values close to the upper end of the FFR uncertainty range.

Example\#1: [stenotic proximal LAD]-[stenotic remainder of LAD]-[stenotic

normalD ${ }_{1}$ ]

In this example LMCA is taken to be stenosis-free so that the aortic pressure is maintained all the way to the beginning of LAD.

The 'mother'-'daughter' configuration can be described in Figure $\mathbf{1}$ by an equivalent 3-artery configuration:

[stenotic proximal LAD] - [stenotic remainder of $\mathrm{LAD}]$ - [stenotic normalD $\left.{ }_{1}\right]=$

$=[$ Artery 1] $-[$ Artery 2] $-[$ Artery 3]

The measured intracoronary mean pressures (Figure 1) are taken to be the following:

$$
\begin{aligned}
& P_{a}=100 \mathrm{mmHg} \\
& \mathbf{P}_{\mathbf{p}}=90 \mathrm{mmHg} \\
& \mathbf{P}_{2 d}=85 \mathrm{mmHg} \\
& \mathbf{P}_{3 d}=80 \mathrm{mmHg}
\end{aligned}
$$

As a first step, the current $\mathrm{FFR}^{\text {true }}$ values of arteries 1, 2 and 3 (see Figure 1) in the limit $1 \ll \delta$ will be calculated: 
$\operatorname{FFR}^{\text {true }}(1)=1 /\left[\left(\mathbf{P}_{\mathbf{a}}-\mathbf{P}_{\mathbf{p}}\right) / \mathbf{P}_{\mathbf{2 d}}+1\right]=0.89 \quad($ see $(8))$

$\operatorname{FFR}^{\text {true }}(2)=\mathbf{P}_{\mathbf{2 d}} / \mathbf{P}_{\mathbf{p}}=0.94 \quad($ see $(2))$

$\operatorname{FFR}^{\text {true }}(3)=\mathbf{P}_{\mathbf{3 d}} / \mathbf{P}_{\mathbf{p}}=0.89 \quad($ see $(3))$

The FFR ${ }^{\text {real }}$ numerical values for each of the arteries of Figure 1 can be also calculated:

$\operatorname{FFR}^{\text {real }}(1) \approx 0.85 \quad($ see $(9))$

$1<<\delta$

$\mathrm{FFR}^{\text {real }}(2) \approx 0.85 \quad($ see $(5))$

$1<<\delta$

$\mathrm{FFR}^{\text {real }}(3) \approx 0.80 \quad($ see $(6))$

$1<<\delta$

Note that $\operatorname{FFR}^{\text {real }}(1) \approx \mathrm{FFR}^{\text {real }}(2) \approx 0.85$ which is also equal to the FFR ${ }^{\text {real }}\left(\right.$ 'mother') $\approx \mathrm{FFR}^{\text {true }}$ ('mother') $\approx \mathbf{P}_{2 \mathbf{d}}$ / $\mathrm{P}_{\mathrm{a}}=85 / 100=0.85$ by the basic FFR method.

All FFR ${ }^{\text {real }}$ values are in the proximity of the upper end of the FFR uncertainty range, so it may be beneficial to exercise a revascularization option. The revascularization strategy can be devised by taking a good look at expressions (10), (11) and (12). All 3 expressions have a common denominator; this will be useful for revascularization calculations.

Revascularization is supposed to raise the FFR ${ }^{\text {true }}$ of the revascularized artery to $\mathrm{FFR}^{\text {true }}=1.00$ and by this to improve also its FFR ${ }^{\text {real }}$ (and usually also that of other arteries through stenosis-stenosis interactions). By expressions (10), (11) and (12), revascularization of just Artery 3 would be beneficial only to Artery 3 but not to other arteries. On the other hand, revascularization of Artery 1would improve the FFR ${ }^{\text {real }}$ of each of the arteries, as can be seen from the following values in the mathematical limit $1<<$.

Revascularization Option \#1:

$\operatorname{FFR}^{\text {true }}(1)=1.00 \quad \operatorname{FFR}^{\text {true }}(2)=0.94 \quad \operatorname{FFR}^{\text {true }}(3)=0.89$

The resulting FFR ${ }^{\text {real }}$ values are:

$$
\begin{aligned}
& \operatorname{FFR}^{\text {real }}(1) \approx 0.94 \quad(\text { see }(10)) \\
& 1<<\delta \\
& \operatorname{FFR}^{\text {real }}(2) \approx 0.94 \quad(\text { see }(11)) \\
& 1<<\delta \\
& \operatorname{FFR}^{\text {real }}(3) \approx 0.89 \quad(\text { see }(12)) \\
& 1<<\delta
\end{aligned}
$$

This outcome is very good and there seems to be no room for improvement by revascularizing also Artery $\mathbf{2}$ since FFR ${ }^{\text {true }}$ (2) is already very close to 1.00 . Note also that this is in accordance with the pullback approach of the basic FFR method within the 'mother' artery: the pressure drop over the stenosis in Artery $\mathbf{1}$ is $\Delta \mathbf{P}_{\mathbf{1}}=$ $\mathbf{P}_{\mathbf{a}}-\mathbf{P}_{\mathbf{p}}=100-90=10 \mathrm{mmHg}$ which is greater than the pressure drop over the stenosis in Artery $\mathbf{2}\left(\Delta \mathbf{P}_{2}=\right.$ $\mathbf{P}_{\mathbf{p}}-\mathbf{P}_{\mathbf{2 d}}=90-85=5 \mathrm{mmHg}$ ), giving Artery $\mathbf{1}$ priority for revascularization.

In the next example we'll consider stenotic arteries with FFR ${ }^{\text {real }}$ numerical values close to the lower end of the FFR uncertainty range. The arterial configuration however will be identical to the one in the first example of the present paragraph (Figure 1).

Example\#2:[stenotic proximal LAD]-[stenotic remainder of $\mathrm{LAD}]$-[stenotic normal $\mathrm{D}_{1}$ ]

As in the first example of this section, LMCA is stenosis-free and the aortic pressure is maintained all the way to the beginning of LAD.

As indicated already, this configuration can be described in Figure $\mathbf{1}$ by an equivalent 3-artery configuration:

[stenotic proximal LAD] - [stenotic remainder of LAD $]-[$ stenotic normalD $1=[$ Artery 1] $-[$ Artery 2 $]$ - [Artery 3]

The measured intracoronary mean pressures (Figure 1) are taken to be the following:

$$
\begin{aligned}
& \mathbf{P}_{\mathrm{a}}=100 \mathrm{mmHg} \\
& \mathbf{P}_{\mathbf{P}}=70 \mathrm{mmHg} \\
& \mathbf{P}_{\mathbf{2 d}}=65 \mathrm{mmHg} \\
& \mathbf{P}_{\mathbf{3 d}}=65 \mathrm{mmHg}
\end{aligned}
$$

These data yield the current FFR ${ }^{\text {true }}$ values of arteries 1, 2 and 3 (Figure 1) in the mathematical limit $1<<\delta$ :

$$
\begin{aligned}
& \operatorname{FFR}^{\text {true }}(1)=1 /\left[\left(\mathbf{P}_{\mathbf{a}}-\mathbf{P}_{\mathbf{p}}\right) / \mathbf{P}_{\mathbf{2 d}}+1\right]=0.69 \quad(\text { see }(8)) \\
& \operatorname{FFR}^{\text {true }}(2)=\mathbf{P}_{\mathbf{2 d}} / \mathbf{P}_{\mathbf{p}} \approx 0.93 \quad(\text { see }(2)) \\
& \operatorname{FFR}^{\text {true }}(3)=\mathbf{P}_{\mathbf{3 d}} / \mathbf{P}_{\mathbf{p}}=0.93 \quad(\text { see }(3))
\end{aligned}
$$

The FFR ${ }^{\text {true }}$ values can be used to obtain the FFR ${ }^{\text {real }}$ for each of the arteries of Figure 1:

$$
\begin{aligned}
& \operatorname{FFR}^{\text {real }}(1) \approx 0.65 \quad(\text { see }(10)) \\
& 1<<\delta \\
& \operatorname{FFR}^{\text {real }}(2) \approx 0.65 \quad(\text { see }(11)) \\
& 1<<\delta
\end{aligned}
$$




$$
\begin{aligned}
& \operatorname{FFR}^{\text {real }}(3) \approx 0.65 \quad(\text { see }(12)) \\
& 1<<\delta
\end{aligned}
$$

It is clear that the FFR ${ }^{\text {real }}$ values are quite low and the condition of this arterial configuration is therefore unacceptable.

Note that in this case the Multi-artery FFR method yields $\operatorname{FFR}^{\text {real }}(1) \approx \mathrm{FFR}^{\text {real }}(2) \approx 0.65$ which is equal to the FFR $^{\text {real }}$ ('mother') $\approx$ FFR $^{\text {true }}$ ('mother') $\approx \mathrm{P}_{2 \mathrm{~d}} / \mathrm{P}_{\mathrm{a}}=65 / 100$ $=0.65$ obtained also by the basic FFR method.

By the Multi-artery FFR method, it is justifiable to revascularize Artery $\mathbf{1}$ first because its FFR ${ }^{\text {true }}$ is less than that of Artery $\mathbf{2}$ and therefore there is more to be gained in elevating FFR ${ }^{\text {real }}$ ('mother') by revascularizing Artery 1. By the basic single-artery FFR pullback approach: the pressure drop over the stenosis in Artery $\mathbf{1}$ is $\Delta \mathbf{P}_{\mathbf{1}}=$ $\mathbf{P}_{\mathbf{a}}-\mathbf{P}_{\mathbf{p}}=100-70=30 \mathrm{mmHg}$ which is greater than the pressure drop over the stenosis in Artery $\mathbf{2}\left(\Delta \mathbf{P}_{2}=\right.$ $\mathbf{P}_{\mathbf{p}}-\mathbf{P}_{\mathbf{2 d}}=70-65=5 \mathrm{mmHg}$ ), therefore also by the basic single-artery FFR method, Artery 1 should be revascularized first.

Artery 1 seems to be the 'bottle-neck' of the whole arterial configuration (with FFR ${ }^{\text {true }}(1)=0.69$ ). Therefore, there is a possibility that its revascularization will suffice for resolving the whole stenotic configuration.

Option \#1 - Revascularization of Artery 1:

$\operatorname{FFR}^{\text {true }}(1)$ becomes $\operatorname{FFR}^{\text {true }}(1)=1.00$ and the FFR ${ }^{\text {true }}$ of the other arteries maintain their current values (Figure 1).

The FFR ${ }^{\text {true }}$ values after revascularization of Artery $\mathbf{1}$ are the following:

$\operatorname{FFR}^{\text {true }}(1)=1.00 \quad \operatorname{FFR}^{\text {true }}(2) \approx 0.93 \quad \operatorname{FFR}^{\text {true }}(3)=0.93$

Using these FFR ${ }^{\text {true }}$ values, the obtained FFR ${ }^{\text {real }}$ values are the following (Figure 1):

$$
\begin{aligned}
& \operatorname{FFR}^{\text {real }}(1) \approx 0.93 \quad(\text { see }(10)) \\
& 1<<\delta \\
& \operatorname{FFR}^{\text {real }}(2) \approx 0.93 \quad(\text { see }(11)) \\
& 1<<\delta \\
& \operatorname{FFR}^{\text {real }}(3) \approx 0.93 \quad(\text { see }(12)) \\
& 1<<\delta
\end{aligned}
$$

The outcome is very good for all arteries. Therefore there is no need for further revascularizations. Note that as in the previous example, the condition of Artery $\mathbf{3}$ has been improved indirectly (through inter-arterial interaction) by revascularizing Artery $\mathbf{1}$.

\section{Discussion}

It is important to note that despite its superiority over the basic FFR method in the stenosis severity assessment of complex arterial configurations in MVD cases [79], the multi-artery FFR method does not contest the clinical findings (FFR treatment-decision ranges) of the basic FFR method with regard to a single stenotic artery. The known FFR treatment criteria ranges apply to each artery of stenotic 2 or 3-artery configurations but they apply to FFR ${ }^{\text {real }}$ of each artery of a configuration, not to its FFR ${ }^{\text {true }}$. However, because of the stenosis-stenosis interactions between the arteries of the configuration, note that the FFR ${ }^{\text {real }}$ of an artery can be improved by revascularizing another artery of the configuration. This article applies also to the instantaneous wave-free ratio (iFR) method [10] and any other FFR-oriented method but it does not imply that the FFR method is the best diagnostic method in cases of coronary artery disease (CAD) under all circumstances.

The Multi-artery FFR method has the capability to resolve all-stenotic 3-artery configurations of coronary arteries [9] in the PCI practice. There is however a difference between the resolution of stenotic configurations of sizable arteries (where $\delta$ is taken to be $\delta=1,[7-9]$ ) and configurations in which one of the arteries is small (where $1<<\delta,[8]$ ), like in this article. Note that in some cases the first diagonal artery $D_{1}$ (taken here as an example of a stenotic small side branch) is a sizable artery. Normally however, as in this article, it is a relatively small side branch of the sizable LAD artery. In the present article the Multi-artery FFR method is adapted to resolution of some stenotic 'mother'-'daughter' configurations (e.g. stenotic LAD, preceded by non stenotic LMCA, and stenotic normal side branch $\mathrm{D}_{1}$ ). The stenoses in the 'mother' artery in this article precede and follow the split-off point of the stenotic 'daughter' artery. The stenotic 'mother''daughter' configuration is resolved by applying the Multi-artery FFR method to an equivalent 3-artery configuration (see Example\#1, Example\#2 and Figure 1).

The stenotic 'mother'-'daughter' configuration that is dealt with in this article is a particular configuration in which one artery ('daughter' side branch) has a negligible effect on the other ones. This is why in this case the basic single artery FFR method can be a substitute for the Multi-artery FFR method in the resolution of this configuration. The resolution by the basic single-artery FFR method is accomplished after at most 3 pullback rounds (including one for the side branch). 
The examples in the article have been intentionally chosen to demonstrate also the possible differences (before revascularization) between $\mathrm{FFR}^{\text {true }}$ and FFR $^{\text {real }}$ of an artery near the upper and lower edges of the FFR uncertainty range (intermediate stenosis severity), FFR ${ }^{\text {true }}-$ FFR $^{\text {real }}-0.1$ and FFR ${ }^{\text {true }}-$ FFR $^{\text {real }}$ - 0.3 respectively (see Example\#1 and Example\#2 in paragraph 3. Results). The Multi-artery FFR method provides the PCI practitioner with the precise current status of the 'mother'-'daughter' configuration (namely FFR $^{\text {rue }}$ and FFR ${ }^{\text {real }}$ of each artery of the equivalent configuration in Figure 1) [9]. The examples show that one of the great advantages of the Multi-artery FFR method is its capability to predict the outcome of all optional revascularizations (after just one pullback round for each artery) that can be useful if the present status of the arterial configuration is not satisfactory. The calculations are simple and can be performed by the practitioner in real time during the PCI procedure.

To conclude, a concise guide for the application of the multi-artery FFR method will now be given. The

\section{References}

1. Sarno G, Lagerqvist B, Frobert O, et al. Lower risk of stent thrombosis and restenosis with unrestricted use of 'newgeneration' drug- eluting stents: a report from the nationwide Swedish Coronary Angiography and Angioplasty Registry (SCAAR). Eur Heart J. 33: 606-613 (2012).

2. Dangas GD, Claessen BE, Caixeta A, et al. In-Stent Restenosis in the Drug-Eluting Stent Era. J Am Coll Cardiol. 56: 1897-907 (2010).

3. Iakovou I, Schmidt T, Bonizzoni E, et al. Incidence, Predictors, and Outcome of Trombosis After Successful Implantation of Drug- Eluting Stents. JAMA. 293: 2126-2130 (2005).

4. Fornell D. Despite Potential Impact,Use Remains Low for Fractional Flow Reserve (FFR). Diagn Interv Cardiol. March 6, 2013.

5. Tonino PAL, De Bruyne B, Pijls NHJ, et al. Fractional Flow Reserve versus angiography for guiding Percutaneous Coronary Intervention. N Engl J Med. 360: 213-224 (2009). application of the Multi-artery FFR to stenotic 'mother''daughter' configurations shown in this article can be used by the PCI practitioner in real time during the PCI procedure by exploring the present status of the equivalent stenotic 3-artery configuration (Figure 1). The process works out as following: Firstly the current FFR true and $\mathrm{FFR}^{\text {real }}$ of each artery are obtained from the measured intracoronary pressures (see expressions (2), (3), (5), (6), (8), (9)). Then, if FFR ${ }^{\text {real }}$ of some artery is not satisfactory, revascularization options are explored through expressions (10), (11) and (12). Using these expressions, one puts $\mathrm{FFR}^{\mathrm{true}}=1.00$ for a revascularized artery while any other artery that does not undergo revascularization retains the current FFR $^{\text {true }}$ that was initially calculated from the measured intracoronary pressures. This eventually yields the optimal resolution of the 'mother'-daughter' configuration. As indicated already, in this particular case in which one artery (the 'daughter' side branch) has a negligible effect on all other members of the configuration, the optimal resolution can be reached also by using the basic single-artery FFR method.
6. Nunen LX, Zimmermann FM, Tonino PAL, et al. Fractional flow reserve versus angiography for guidance of $\mathrm{PCI}$ in patients with multivessel coronary artery disease (FAME): 5 -year followup of a randomized controlled trial. Lancet. 386: 1853-1860 (2015).

7. Yaeger IA. A multi-artery Fractional Flow Reserve (FFR) approach for handling coronary stenosis-stenosis interaction in the multi-vessel disease (MVD) arena. Int J Cardiol. 203: 807815 (2016).

8. Yaeger IA. Practicability of Multi-artery Fractional Flow Reserve (FFR) Method in the Assessment of Some Stenotic Coronary Artery Configurations in Percutaneous Coronary Intervention (PCI) Procedures. Interv Cardiol J. 3: 3 (2015).

9. Yaeger IA. Resolution of All-stenotic 3-artery Configurations in Percutaneous Coronary Intervention (PCI) Procedures by The Multi-artery Fractional Flow Reserve (FFR) Method. Cardiovasc Investig. 1: 2 (2017).

10. Nijjer SS, Sen S, Petraco R, et al. Advances in coronary physiology. Circ J. 79(6): 1172-84 (2015). 